\title{
Environmental Sustainability in the Synthesis and Characterization of Hybrid/Composite Nanomaterials
}

\author{
Maria Giulia Faga $\mathbb{D}^{1},{ }^{1}$ Roberto Nisticò $\mathbb{D},{ }^{2}$ Jana Kukutschova, ${ }^{3}$ Giuliana Magnacca $\mathbb{D},{ }^{4}$ \\ and Luciano Carlos $\mathbb{D D}^{5}$ \\ ${ }^{1}$ CNR-IMAMOTER, Torino, Italy \\ ${ }^{2}$ Politecnico di Torino, Torino, Italy \\ ${ }^{3}$ VSB-Technical University of Ostrava, Ostrava, Czech Republic \\ ${ }^{4}$ Università degli Studi di Torino, Torino, Italy \\ ${ }^{5}$ Instituto de Investigación y Desarrollo en Ingeniería de Procesos, Biotecnología y Energías Alternativas, PROBIEN (CONICET- \\ UNCo), Neuquén, Argentina
}

Correspondence should be addressed to Maria Giulia Faga; m.faga@imamoter.cnr.it

Received 27 November 2018; Accepted 27 November 2018; Published 9 December 2018

Copyright (c) 2018 Maria Giulia Faga et al. This is an open access article distributed under the Creative Commons Attribution License, which permits unrestricted use, distribution, and reproduction in any medium, provided the original work is properly cited.

The guest editors for this special issue are pleased to welcome contributions from researchers studying hybrid/composite nanomaterials reporting significant advancements in terms of environmental friendliness and sustainability in their preparation and characterization methods. Manuscripts selected for this special issue deal with the development of alternative/innovative synthetic routes for the production of nanocomposites and/or hybrid materials, with proven or expected reduction of the overall environmental impact as well as the production of biocompatible components or showing improved sustainability.

Four articles focused on the use of (eventually magnetic) nanomaterials for the remediation of contaminated media via either photocatalysis or adsorption mechanisms. M. Etay et al. evaluated the photocatalytic properties of bare, Agdoped, and $\mathrm{Mn}$-doped $\mathrm{ZnO}$ nanoparticles prepared via a coprecipitation method by monitoring the light-induced decolorization of methyl violet aqueous solutions. P. Niu et al. synthetized bifunctional films of Keggin-type polyoxometalates and $\mathrm{TiO}_{2}$ onto $\mathrm{Fe}_{3} \mathrm{O}_{4} @ \mathrm{SiO}_{2}$ microspheres using a layer-by-layer method and tested their photocatalytic efficiencies in the photo-induced degradation of methyl orange. X. T. Chu et al. studied the performances of magnetic nanocomposites made by polyaniline-coated magnetite nanoparticles for the removal of As(III) from simulated contaminated aqueous medium via adsorption. Lastly, B. Abebe and H. C. Ananda Murthy reported the production of magnetic $\mathrm{TiO}_{2}-\mathrm{Fe}_{2} \mathrm{O}_{3}$ binary oxides and their adsorption performances against $\mathrm{Pb}(\mathrm{II})$ ions from aqueous solution.

The production and testing of magnetic materials for other purposes were also evaluated by Y. Jiraskova et al., who analyzed the structural and magnetic properties of magnetically modified clays (i.e., $\mathrm{Fe}_{3} \mathrm{O}_{4}$ introduction into both montmorillonite and vermiculite) and compared to the unmodified clay minerals.

The other two articles devoted to the improvement of mechanical properties of nanomaterials. J. L. Orellana et al. evaluated the effect of introducing surfactants as compatibilizers in blends of surface-modified cellulose nanocrystals with a polylactic acid (PLA) matrix produced via film extrusion. K. Dedkova et al. introduced ad hoc prepared kaolin/ $/ \mathrm{TiO}_{2}$ nanocomposites in a commercially available automotive low-steel brake pad, observing an improvement in the wear resistance (and consequently a reduction in both wear rate and particulate emission).

In our opinion, the seven articles forming this special issue are good examples on how the development of advanced nanocomposites is able to both directly and 
indirectly improve the environmental sustainability of products and processes. The editors hope that these scientific contributions will positively stimulate the creativity of researchers to focus their efforts on this very intriguing research field.

\section{Conflicts of Interest}

The editors declare that they have no conflicts of interest regarding the publication of this special issue.

\section{Acknowledgments}

The editors would like to thank all authors for having submitted their manuscripts to this special issue, the editors for having spent their time selecting reviewers and making editorial decisions, and of course the reviewers for providing their expert opinions.

Maria Giulia Faga Roberto Nisticò

Jana Kukutschova Giuliana Magnacca Luciano Carlos 


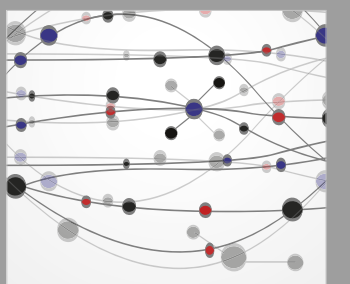

The Scientific World Journal
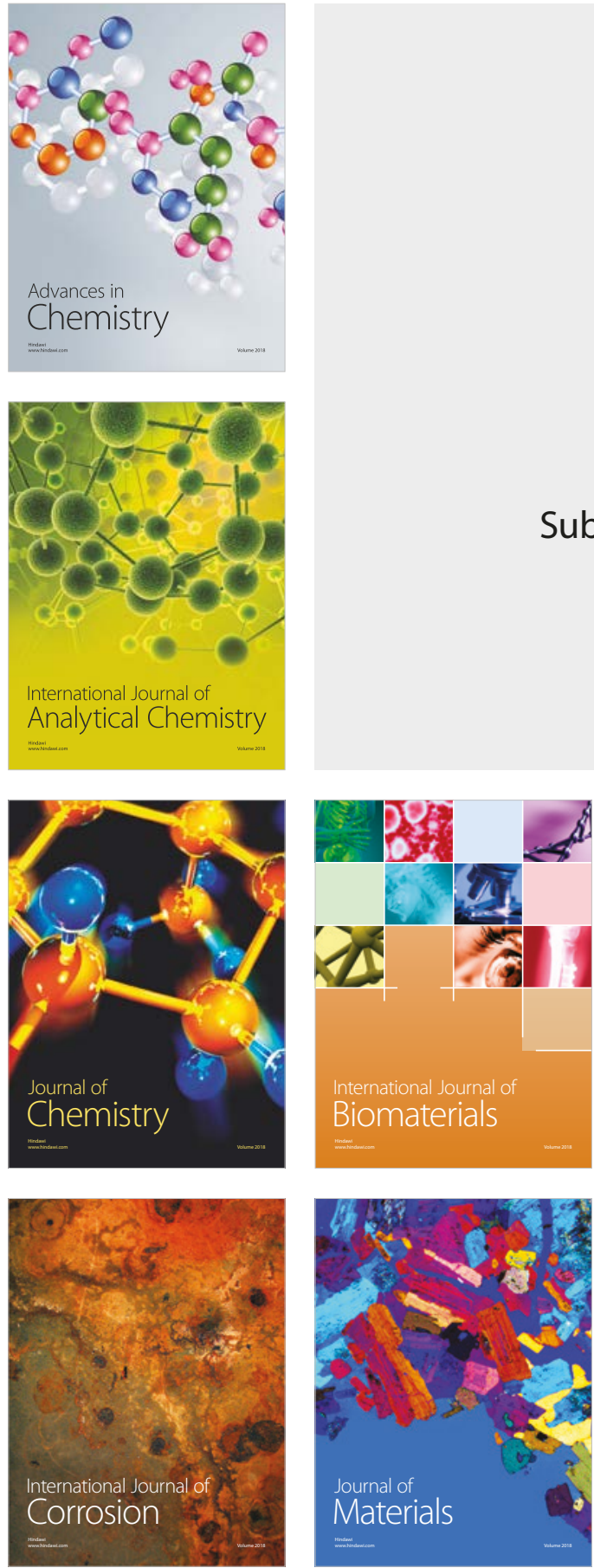

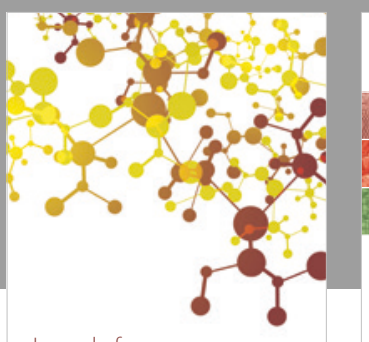

Journal of

Applied Chemistry
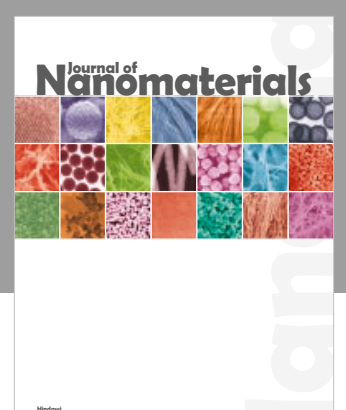

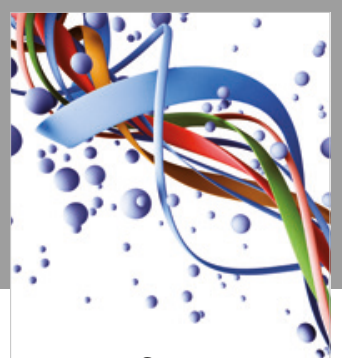

Scientifica

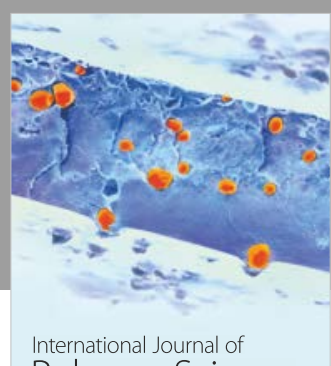

Polymer Science

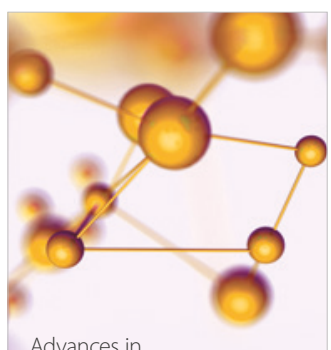

Physical Chemistry
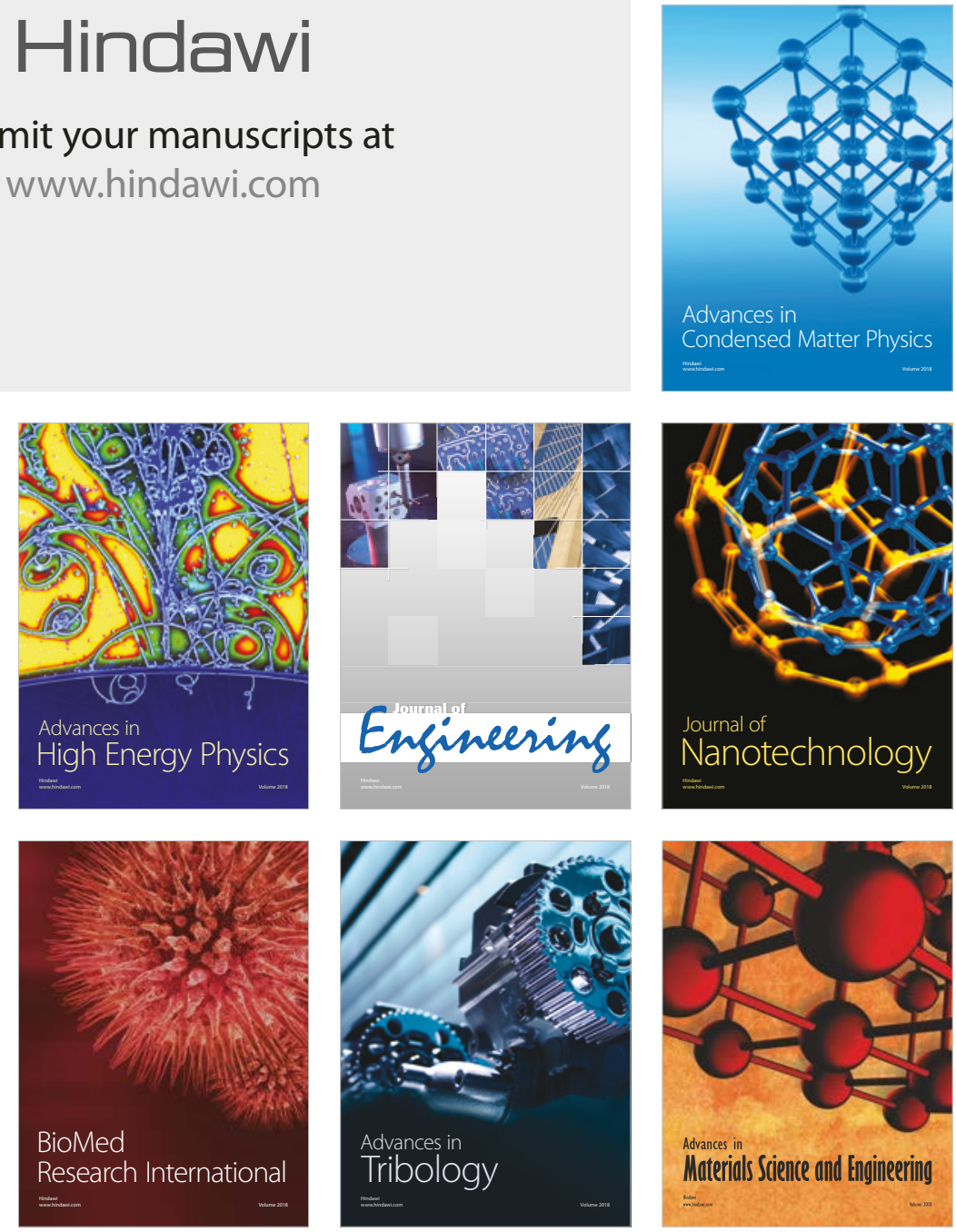